\title{
MICRO-RÉSONATEURS EN ANNEAU POUR LA DÉTECTION ET L'IDENTIFICATION DE POLLUANTS
}

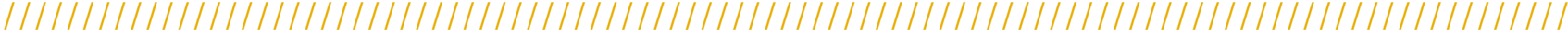 \\ Chi Thanh NGUYEN, Isabelle LEDOUX-RAK* \\ Laboratoire LuMIn, ENS Paris Saclay, CNRS, CentraleSupelec - Université Paris Saclay - 91190 Gif-sur-Yvette, France \\ *Isabelle.ledoux@ens-paris-saclay.fr}
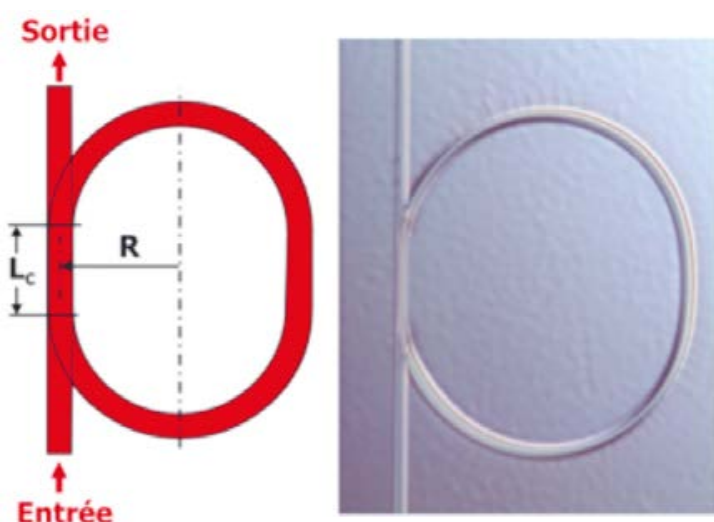

Entrée

Parmi les capteurs optiques visant la détection d'espèces chimiques ou biologiques, les dispositifs basés sur des microrésonateurs présentent un intérêt spécifique: ils n'ont pas besoin de marqueurs fluorescents, et leur fabrication est peu coûteuse. Le présent article décrit un capteur de ce type, basé sur des guides en anneau immergés dans un circuit microfluidique, et donne une illustration de ses performances pour la détection d'ions lourds dans l'eau potable.

\section{https://doi.org/10.1051/photon/202110634}

Article publié en accès libre sous les conditions définies par la licence Creative Commons Attribution License CC-BY (https://creativecommons.org/licenses/by/4.0), qui autorise sans restrictions l'utilisation, la diffusion, et la reproduction sur quelque support que ce soit, sous réserve de citation correcte de la publication originale.

Parmi les solutions proposées, les microrésonateurs présentent un intérêt particulier, du fait de leur grande sensibilité et de leur rapidité de détection d'espèces situées à proximité de leur interface avec le milieu extérieur [1,2]. La détection est basée sur l'interaction entre une onde évanescente du microrésonateur optique et les objets à détecter près de sa surface. La spécificité de la détection est assurée par la fonctionnalisation sur cette surface d'une molécule de reconnaissance spécifique de l'espèce cible.
Diverses géométries de microrésonateurs ont été étudiées (sphères, disques, anneaux) [1]. Dans les deux premiers cas, les modes optiques excités sont des modes dits de galerie, générés par réflexion totale sur l'interface du résonateur avec l'extérieur. Leurs facteurs de qualité peuvent être très élevés, mais le couplage avec un guide permettant d'injecter la lumière est difficile, et peu compatible avec un fonctionnement en phase liquide. En revanche, les microrésonateurs 
à modes guidés (anneaux), de facteurs de qualité un peu plus faibles, se prêtent bien à l'intégration en milieu fluidique.

Un exemple d'un tel capteur, intégré à une cellule opto-fluidique, est présenté ici. Le microrésonateur est fonctionnalisé par des molécules de reconnaissance spécifiques, ce qui permet la détection ultra-sensible et en temps réel de polluants de l'eau potable ou de molécules biologiques.

\section{CONCEPTION DE MICRO- RESONATEURS OPTIQUES}

\section{Principe}

Le principe du fonctionnement d'un capteur optique à base de micro-résonateurs est illustré sur la figure 1.

La capture de l'espèce cible modifie la réponse spectrale du résonateur, ce qui se traduit, lors d'un balayage en longueur d'onde, par un déplacement du pic de résonance $\delta \lambda$, ou par une variation de l'intensité $\Delta \mathrm{I}$ à une longueur d'onde fixe $\lambda_{0}$. La première solution, plus fiable, est la plus largement adoptée, mais elle nécessite une source à longueur d'onde variable avec une excellente résolution spectrale (de l'ordre de $0,1 \mathrm{pm}$ ), ce qui justifie l'utilisation d'un laser à $1,55 \mu \mathrm{m}$ pour les télécommunications optiques. Dans ces conditions, il n'est pas nécessaire d'analyser la largeur spectrale du signal de sortie, puisque la résolution est fournie par la source. En outre, cette longueur d'onde facilite la fabrication de guides optiques monomodes, dont les dimensions transverses sont plus grandes que

\section{Les microrésonateurs à modes guidés (anneaux), de facteurs de qualité un peu plus faibles, se prêtent bien à l'intégration en milieu fluidique.}

pour des longueurs d'onde visibles, les détecteurs restant par ailleurs d'un coût raisonnable.

La seconde solution, moins exigeante en résolution, nécessite une légère accordabilité pour fixer la longueur d'onde de travail au point d'inflexion de la courbe de réponse, où la sensibilité est la plus grande.

\section{Architecture}

Le schéma du microrésonateur est indiqué sur la figure 2. L'architecture de ces dispositifs doit présenter certaines caractéristiques :

Les guides doivent fonctionner en régime monomode, afin de pouvoir facilement isoler un pic de résonance.

- Le couplage entre le guide rectiligne et le microrésonateur doit

\section{Figure 1.}

(a) Vue de dessus d'un microrésonateur en anneau, couplé verticalement à un guide enterré pour assurer l'insertion (Entrée) et l'extraction (Sortie) de l'onde optique. L"onde évanescente est schématisée en jaune. (b) Principe de la capture, par un ligand de reconnaissance greffé à la surface, des espèces cibles qui interagissent avec l'onde évanescente. (c) Principe de la détection des espèces cibles (voir b) par interrogation spectrale ou d'intensité. pouvoir être assuré en utilisant des technologies de fabrication qui n'exigent pas une très haute résolution spatiale comme la gravure à faisceaux d'électrons. Il ne doit pas être perturbé par la présence d'un fluide entre ce guide et le microrésonateur. Ces deux raisons justifient le choix d'une technologie à base de polymères, avec un couplage vertical [1], l'espace (gap) d'épaisseur g entre le guide rectiligne et l'anneau étant occupé par un polymère de bas indice.

La forme de l'anneau est celle d'un « hippodrome» (deux arcs de cercle reliés par deux guides rectilignes), ce qui permet d'ajuster le couplage en fonction de 3 paramètres $\left(R, L_{c}\right.$ et $\mathrm{g}$ ) et pas seulement de $\mathrm{R}$ et de $\mathrm{g}$ comme avec un anneau circulaire.

Ces paramètres permettent d'optimiser la détection chimique ou biochimique en phase liquide, la couche de confinement supérieure étant la solution d'analyte (Fig. 2a). Les matériaux sont des polymères transparents dans le visible et le proche IR, compatibles avec des méthodes de dépôt et de gravure à bas coût [3].

L'optimisation de la géométrie des micro-cavités en forme d'hippodrome (Fig. $2 b$ ) est réalisée par des simulations numériques, autour d'une longueur d'onde de 1,55 $\mu \mathrm{m}$. La couche de confinement inférieure est en silice. Une photo-résine négative commerciale constitue le cœur du guide monomode rectiligne et $\mathrm{du}$ micro-anneau. La couche de confinement supérieure du guide $\bullet \bullet \bullet$

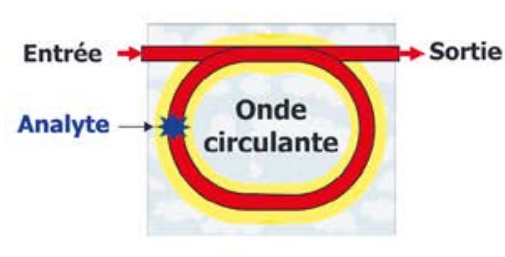

(a)

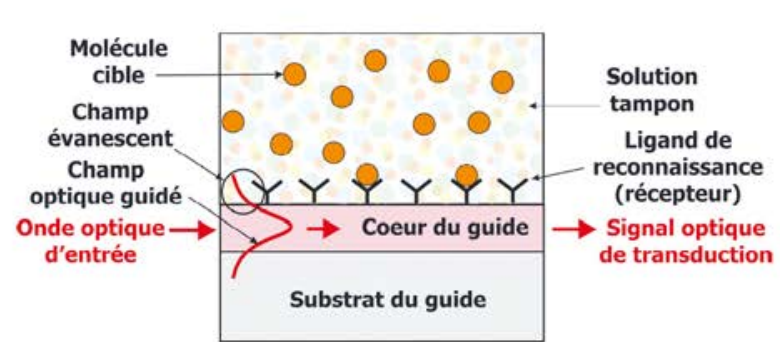

(b)

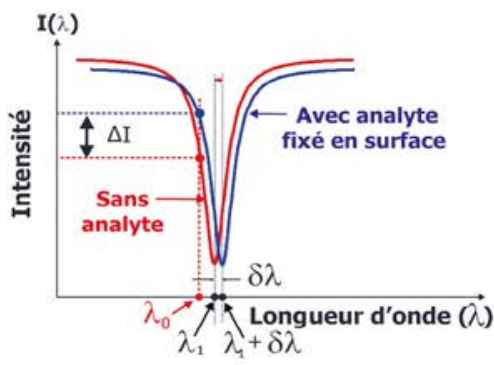




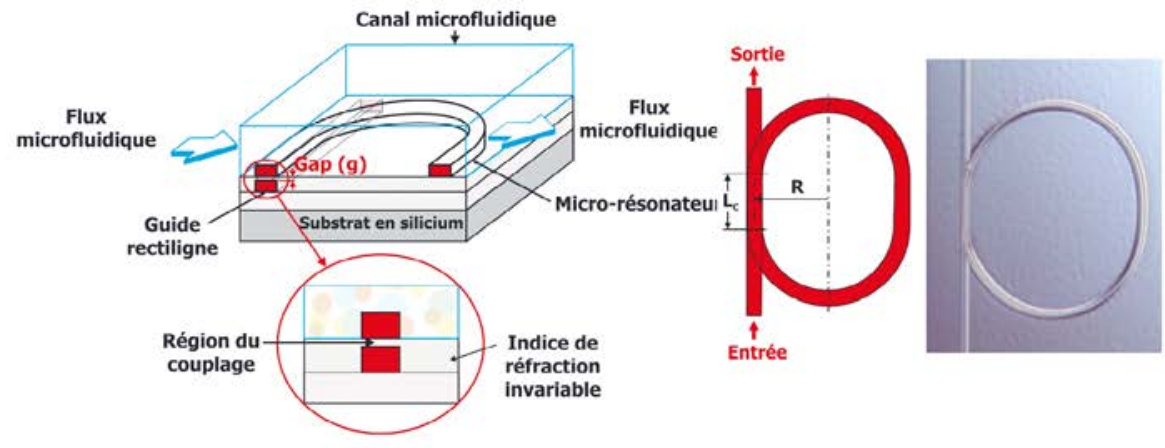

(a)

(b)
Figure 2.

(a) Configuration d'un micro-résonateur en couplage vertical dans une cellule opto-fluidique; (b, à gauche): configuration du couplage entre guide d'injection et microrésonateur ;

(b, à droite): photographie au microscope optique du microrésonateur et de son guide d'injection ; (c) réponse spectrale de ce résonateur illustrant son facteur de qualité.

Figure 3.

Composant optofluidique pour mesures différentielles (a), en multiplexage (b) et en parallèle (c), photographie au microscope optique du composant (d).

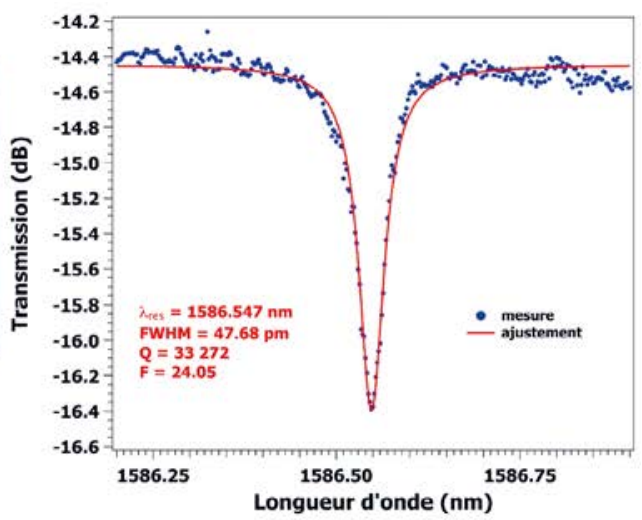

(c) rectiligne constitue aussi la couche polymère fluoré commercial à bas indice, et son épaisseur g peut varier entre $80 \mathrm{~nm}$ et $200 \mathrm{~nm}$. Le rayon $220 \mu \mathrm{m}$, la longueur de couplage $\left(\mathrm{L}_{\mathrm{c}}\right)$ du micro-résonateur de 0 à $220 \mu \mathrm{m}$. La section des guides est d'environ $10 \mu \mathrm{m}^{2}$.

\section{Mesures différentielles}

Pour s'affranchir des erreurs et dérives systématiques de mesure (variation de température ou autres paramètres environnementaux) et pour optimiser l'extraction du signal de mesure d'un bruit de fond d'origines diverses, on développe un montage de mesure différentielle, comprenant deux microrésonateurs immergés dans un ou deux canaux microfluidiques (Fig. 3a). Dans la cellule dite de référence, le micro-résonateur n'est pas fonctionnalisé pour ne pas détecter en surface la molécule cible. En revanche, dans la cellule de mesure, le micro-résonateur est fonctionnalisé afin de détecter à sa surface l'espèce cible. Les deux cellules sont exposées à la même solution d'analyte, à la même pression et au même débit de flux micro-fluidique. Elles subissent les mêmes perturbations physiques durant la mesure ; mais la molécule cible n'est détectée par la molécule de reconnaissance que dans la cellule de mesure.

On peut utiliser la même architecture pour réaliser des mesures

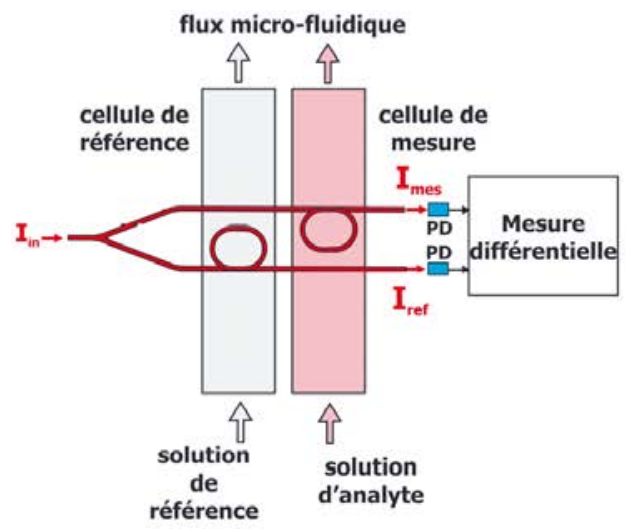

(a)

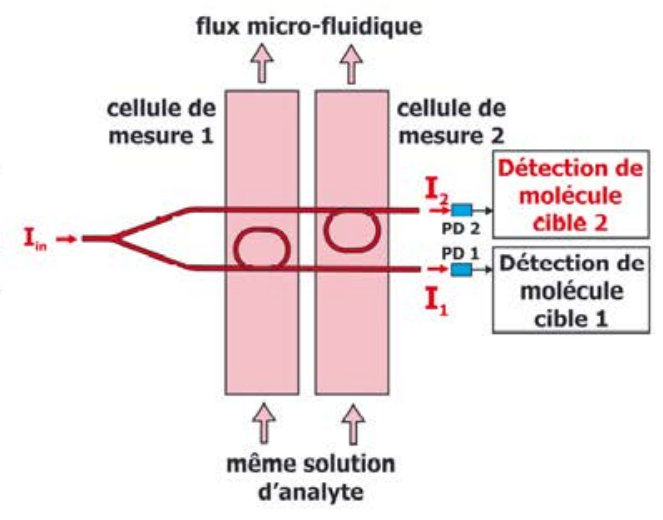

(b)

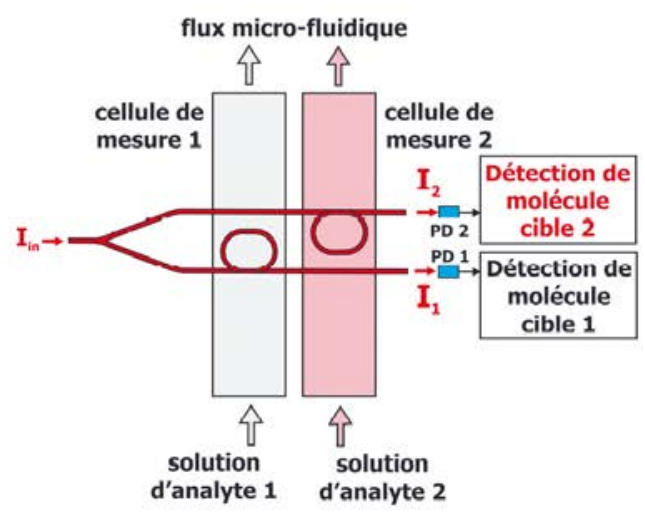

(c)

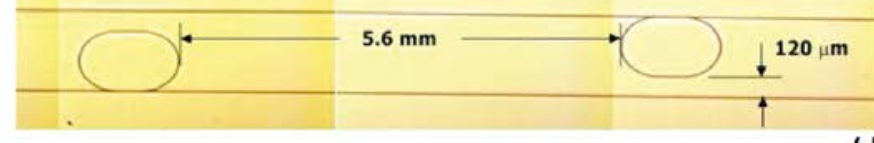


Ce facteur de qualité, mesuré avec l'eau dé-ionisée comme couche de confinement supérieure, est le plus élevé obtenu pour des micro-résonateurs réalisés en polymère jusqu'à présent à notre connaissance [1]. en multiplexage ou en parallèle (Figs. 3b et c). Si l'on veut détecter simultanément deux molécules cibles 1 et 2 dans une même solution d'analyte, chaque micro-résonateur doit être fonctionnalisé par un ligand spécifique de la molécule cible correspondante. Les deux micro-résonateurs sont immergés dans la même solution d'analyte, circulant au même régime de flux microfluidique (Fig. $3 \mathrm{~b}$ ).

\section{FABRICATION DE LA}

\section{CELLULE OPTO-FLUIDIQUE}

Les guides et micro-résonateurs sont fabriqués en utilisant une combinaison de photolithographie UV conventionnelle pour la photo-résine constituant les guides et d'une gravure sèche RIE (Reactive Ion Etching) pour contrôler l'épaisseur du gap en polymère de bas indice.

La cellule opto-fluidique du capteur est fabriquée par moulage d'un «capot » micro-fluidique en PDMS (polydiméthylsiloxane) qui est ensuite intégré au circuit optique. Pour injecter la solution d'analyte et le liquide de référence, ce circuit micro-fluidique comprend un ou plusieurs réservoirs, alimentés par une station micro-fluidique. Le montage d'intégration du capot micro-fluidique sur le circuit optique exploite l'élasticité du PDMS, qui permet l'adhésion sur le circuit optique par simple application d'une pression sur le capot microfluidique, sans nécessiter de collage. Enfin, la cellule est montée sur un support thermostaté par des éléments Peltier, qui contrôlent sa température à $0,1 \mathrm{~K}$ près afin d'assurer la stabilité temporelle de la réponse du capteur. Ce montage opto-fluidique est ensuite monté sur le banc de mesure optique. L'injection du signal optique à l'entrée ainsi que sa récupération à la sortie du micro-résonateur sont assurées par des objectifs de microscope ou des fibres optiques.

\section{DÉTECTION DE POLLUANTS \\ Performances optiques du micro-résonateur}

En balayant en longueur d'onde $\lambda$ le spectre de la source laser d'injection, on enregistre la variation d'intensité (transmise et/ou réfléchie) issue du micro-résonateur en fonction de $\lambda$, à $22^{\circ} \mathrm{C}$ (Fig. 2c). La meilleure performance est obtenue pour $\mathrm{R}=180 \mu \mathrm{m}, \mathrm{L}_{\mathrm{c}}=120 \mu \mathrm{m}$, autour de 1585,5 $\mathrm{nm}$. L'intervalle spectral libre est de $1,15 \mathrm{~nm}$, ce qui correspond à un facteur de qualité de 33200 et une finesse de 24. Ce facteur de qualité, mesuré avec l'eau dé-ionisée comme couche de confinement supérieure, est le plus élevé obtenu pour des microrésonateurs réalisés en polymère jusqu'à présent à notre connaissance [1].

\section{Interrogation optique du capteur opto-fluidique}

La présence d'espèces cibles dans l'analyte se traduit par le décalage spectral $\delta \lambda$ du pic de résonance du micro-résonateur par rapport à une solution sans espèce cible (Fig. 1c). En mesurant $\delta \lambda$, on peut déterminer la quantité de biomolécules cibles en solution, à condition de disposer d'une source laser de très grande résolution spectrale $(0,1 \mathrm{pm})$, ce qui permet d'obtenir, grâce à un traitement approprié des signaux optiques, des limites de détection dépassant celles de l'état de l'art. Un avantage important de ce dispositif réside dans la possibilité d'enregistrer sa réponse optique pour deux polarisations perpendiculaires, ce qui fournit de précieuses informations sur l'orientation des molécules attachées en surface.
Wavefront sensor

based platform for

Freeform optics

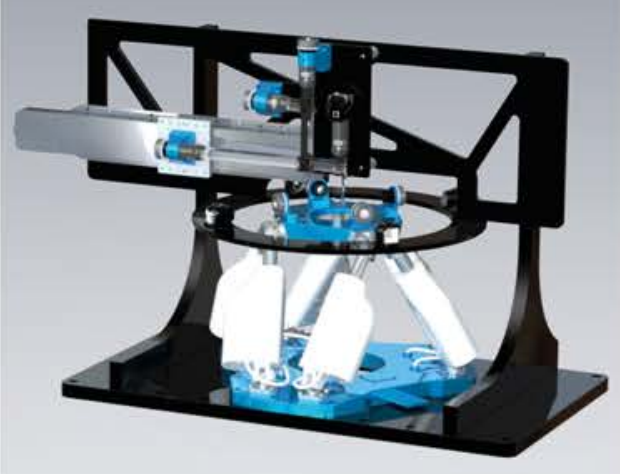

High spatial resolution (down to $30 \mu \mathrm{m}$ sampling)

High accuracy (20 nm RMS)

High dynamic range

\section{Large sample diameter (up to $100 \mathrm{~mm}$ )}

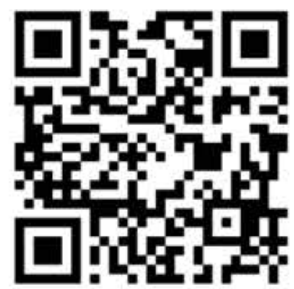

contact@imagine-optic.com

www.imagine-optic.com www.ioweb.store

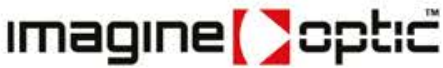



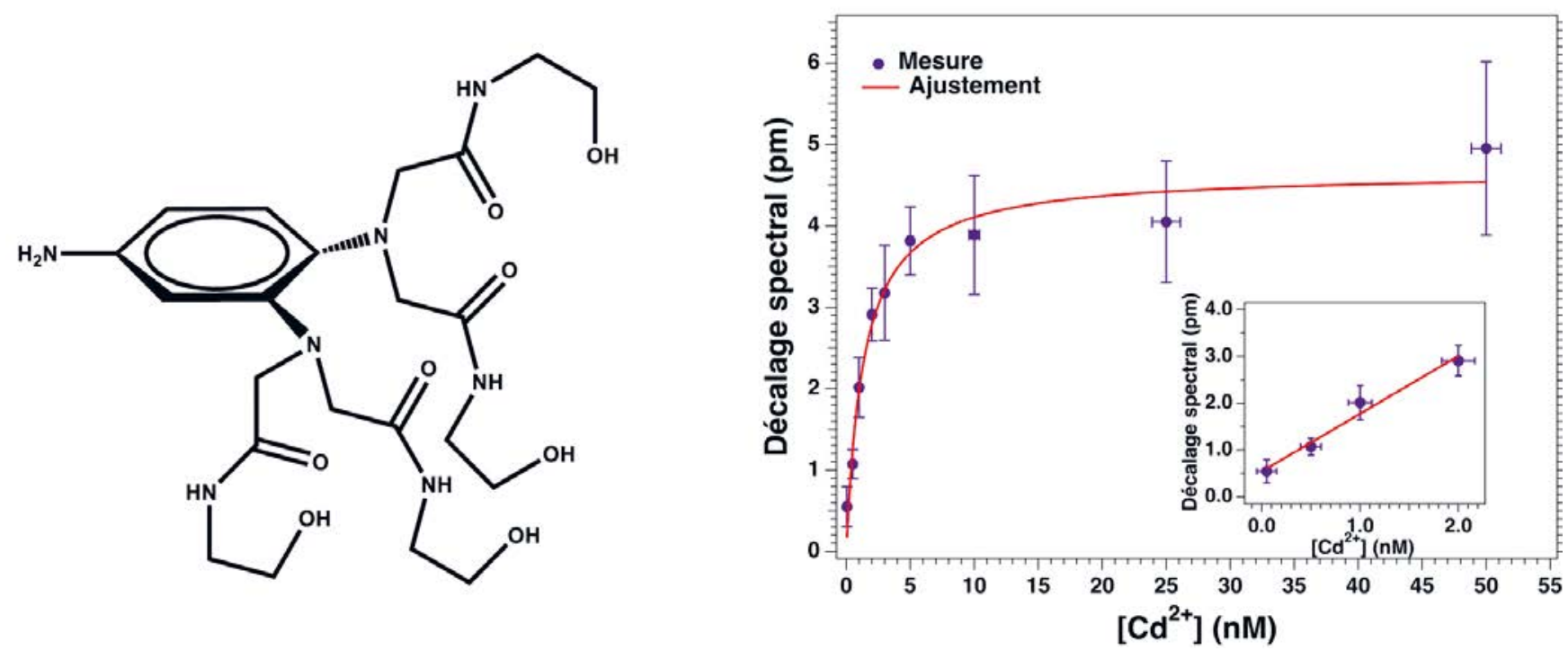

\section{RÉSULTATS : DETECTION}

\section{D'IONS CADMIUM DANS L'EAU}

Un exemple de détection de polluant dans l'eau potable a été mis en évidence en greffant à la surface du micro-anneau un ligand spécifique (synthétisé au laboratoire PPSM de l'ENS Paris Saclay) pour la détection d'ions cadmium $\mathrm{Cd}^{2+}$ (Fig. 4), ions « lourds » toxiques à l'instar du plomb ou du mercure. Le groupe amino $\left(\mathrm{NH}_{2}\right)$ du ligand sert à le fixer sur la surface activée du microrésonateur.

La figure 4 représente la courbe de réponse du capteur opto-fluidique en fonction de la concentration en ions cadmium dans l'eau dé-ionisée. La limite de détection est de $(0,13 \pm 0,03) \mathrm{nM}$, soit $(14,8 \pm 0,07) \mathrm{ng} / \mathrm{L}$. Pour étudier l'interférence des impuretés contenues dans l'eau potable de la ville sur la détection surfacique d'ions cadmium, la détection du cadmium dans l'eau de ville a été démontrée. Même dans ce cas, la limite de détection est peu dégradée, passant de 0,13 à $0,17 \pm 0,04 \mathrm{nM}$, soit $18,3 \pm 4,4$ ng/L [4].

\section{CONCLUSION}

Ce type de capteur illustre les performances des dispositifs optiques de type microrésonateur pour la détection spécifique de polluants dans l'eau potable, par comparaison avec un capteur fluorométrique microfluidique [5]. La limite de
Figure 4.

(Gauche) : ligand pour la détection spécifique $\mathrm{d}^{\prime}$ ions $\mathrm{Cd}^{2+}$. (Droite) : Courbe d'étalonnage de la détection spécifique des ions cadmium dans l'eau dé-ionisée. L'encart montre la partie linéaire de la courbe d'étalonnage utilisée pour évaluer la sensibilité du capteur.

\section{Leurs performances sont} du même ordre de grandeur que celles d'autres méthodes optiques, mais avec une plus grande rapidité de détection, une plus grande sensibilité dans l'eau du robinet, et la possibilité d'étudier les changements d'orientation de molécules greffées en surface en fonction de différents paramètres détection est 25 fois plus faible que la concentration maximale européenne autorisée dans l'eau potable $(0,45 \mu \mathrm{g} / \mathrm{L})$. Leurs performances en termes de limite de détection sont du même ordre de grandeur que celles d'autres méthodes optiques comme la résonance plasmon de surface, mais avec une plus grande rapidité de détection, une plus grande sensibilité dans l'eau du robinet, et la possibilité d'étudier les changements d'orientation de molécules greffées en surface en fonction de différents paramètres (température, composition du solvant...). Il est bien sûr possible d'échanger le ligand chélatant par un autre présentant des affinités pour d'autres objets, ions ou molécules. Enfin, ce type de capteur optofluidique peut être adapté en un capteur portable ou transportable pour réaliser des mesures in situ dans un environnement naturel.
RÉFÉRENCES

[1] F. Vollmer, L. Yang, Nanophotonics 1, 267 (2012)

[2] C. Ciminelli, C.M. Campanella, F. Dell'Olio et al., Prog. Quantum. Electron. 37, 51 (2013)

[3] C. Delezoide, M. Salsac, J. Lautru et al., IEEE Phot. Technol. Lett. 24, 270 (2012)

[4] D Chauvin, J. Bell, I. Leray et al., Sens. Actuator B-Chem. 280, 77 (2019)

[5] H. Zhang, D. Faye, J.P. Lefèvre et al., Microchem. J. 106, 167-173 (2013) 